\title{
A Feedback Control Scheme for Multiple Independent Dynamic Non-point Agents
}

\author{
Dimos V. Dimarogonas and Kostas J. Kyriakopoulos
}

Control Systems Laboratory, Mechanical Eng. Dept.,

National Technical University of Athens,

9 Heroon Polytechniou Street, Zografou 15780, Greece

EMAIL: ddimar@mail.ntua.gr, kkyria@central.ntua.gr

Phone: ++30-210-772-3595,++30-210-772-1726,

Fax: $++30-210-772-3657$

\begin{abstract}
The Decentralized Navigation Functions'(DNF) based methodology, established in our previous work([8],[17]) on multi-agent systems with kinematic models of motion, is extended to the case where dynamic models of motion for both holonomic and nonholonomic agents must be treated. The proposed control scheme guarantees collision avoidance and global convergence of the multi-agent team to the desired goal configuration. The volume of each agent is taken into account and non-point models are considered. The satisfaction of the imposed system specifications under the proposed control scheme are verified and depicted through non-trivial computer simulations.
\end{abstract}




\section{Introduction}

Decentralized Navigation of multiple agents is a field that has recently gained increasing attention in both the robotics and the control communities, due to the need for autonomous control of more than one mobile vehicles in the same workspace. In previous work, the authors extended the seminal work of Koditscheck and Rimon [12] to decentralized concepts. The work in [12] involved navigation of a single point robot in an environment with static obstacles. In [16] this method was successfully extended to take into account the volume of each robot in a centralized multi-agent scheme, while a decentralized version of this work has been presented by the authors in [8],[5],[29],[4] for multiple holonomic and in [17] for multiple nonholonomic agents. In these papers, the volume of each agent was taken into account, and non-point, sphere world agents were considered.

The mathematical models of each agents' motion in these papers were purely kinematic. In practice however, second order models approximate more accurately the real dynamics of multi-agent systems due to the fact that moving vehicles are usually controlled through their acceleration. It is therefore both motivating and natural to extend the methodology introduced in $[8],[17],[5]$ to the case of multi-agent systems with holonomic and nonholonomic dynamics. It should be noted that the results of the holonomic case were partially presented in [6], while the results of the nonholonomic case were partially presented in [7].

Navigation of multiple point agents using decentralized navigation functions was used recently in [28]. Collision avoidance issues for multiple independent point agents were also addressed in [10],[27],[21]. Decentralized Navigation of sphere world agents are considered in the probabilistic setup of [22]. In addition, there is a vast recent literature on cooperative and formation control of multiple point agents that does not take collision avoidance 
issues into account; results include [3],[11], [20],[14],[23] [15],[13]. In this paper, we provide control laws for multiple independent non-point, sphere-world agents, with dynamic models of motion.

The rest of the paper is organized as follows: section 2 provides a revision of the decentralized navigation functions' approach for multiple agents with first order kinematics [5]. The proposed control strategy for a multi-agent system with double integrator dynamics is presented in section 3. The nonholonomic counterpart is found in section 4. The collision avoidance and global convergence properties of the proposed scheme are verified through non-trivial computer simulations in section 5 , while in section 6 conclusions and issues for future research are discussed.

\section{Decentralized Navigation Functions}

In this section, we review the decentralized navigation function method used in [8] for the case of multiple holonomic agents with single integrator kinematics. A detailed description of the concepts presented in the following paragraphs can be found in [5].

Consider a system of $N$ agents operating in the same workspace $W \subset \mathbb{R}^{2}$. Each agent $i$ occupies a disk: $R=\left\{q \in \mathbb{R}^{2}:\left\|q-q_{i}\right\| \leq r_{i}\right\}$ in the workspace where $q_{i} \in \mathbb{R}^{2}$ is the center of the disk and $r_{i}$ is the radius of the agent. In this section, the motion of each agent is assumed purely kinematic and is described by $\dot{q}_{i}=v_{i}$. The configuration space is spanned by $q=\left[q_{1}, \ldots, q_{N}\right]^{T}$. The desired destinations of the agents are denoted by the index $d: q_{d}=$ $\left[q_{d 1}, \ldots, q_{d N}\right]^{T}$. Figure 1 shows a three-agent conflict situation:

Navigation functions are real valued maps realized through cost functions, whose negated gradient field is attractive towards the goal configuration and repulsive with respect to obstacles. It has been shown by Koditscheck and Rimon that almost global navigation is possible since a smooth vector field 


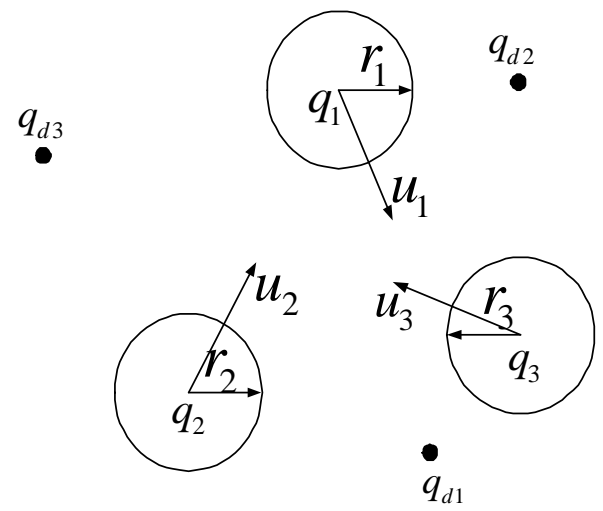

Figure 1: A conflict scenario with three agents.

on any sphere world with a unique attractor, must have at least as many saddles as obstacles ([12]).

A decentralized navigation function $\varphi_{i}$ is defined as $([8],[5])$ :

$$
\varphi_{i}=\frac{\gamma_{d i}+f_{i}}{\left(\left(\gamma_{d i}+f_{i}\right)^{k}+G_{i}\right)^{1 / k}}
$$

The term $\gamma_{d i}=\left\|q_{i}-q_{d i}\right\|^{2}$ is the squared metric of the agent's $i$ configuration from its desired destination $q_{d i}$. The exponent $k$ is a scalar positive parameter. The function $G_{i}$ expresses all possible collisions of agent $i$ with the others, while $f_{i}$ guarantees that the $\varphi_{i}$ attains positive values in proximity situations even when $i$ has already reached its destination.

A detailed presentation of each of the terms defining our Decentralized Navigation Function can be found in [5]. The control strategy for each agent in [5] is given by $v_{i}=-K_{i} \frac{\partial \varphi_{i}}{\partial q_{i}}$, where $K_{i}$ is a positive gain.

A key feature of navigation functions and in particular, DNF's, is that their negative gradient motion is repulsive with respect to the boundary of the free space. The free space for each agent is defined as the subset of $W$ which is free of collisions with the other agents. Hence collision avoidance is reassured. For further information regarding terminology and the single integrator case the reader is referred to $([16],[8],[5])$. 


\section{The Case of double integrator dynamics}

For the case of single integrator kinematics the control law had the simple form $v_{i}=-K_{i} \frac{\vartheta \varphi_{i}}{\vartheta q_{i}}$.

In this section, we assume that the spherical agents' motion is described by the double integrator:

$$
\begin{aligned}
& \dot{q}_{i}=v_{i} \\
& \dot{v}_{i}=u_{i}
\end{aligned}, i \in\{1, \ldots, N\}
$$

Specifically, we consider in this section the decentralized conflict avoidance problem for the case of a multi-agent system with double integrator dynamics. The problem that we treat can be stated as follows: "Derive a set of control laws (one for the acceleration of each agent) that drives the team of $N$ agents from any initial configuration to a desired goal configuration avoiding at the same time collisions." We make the following assumptions:

- Each agent has global knowledge of the position and velocity of the others at each time instant.

- Each agent has knowledge only of its own desired destination but not of the others.

- We consider spherical agents.

- The workspace is assumed to be bounded and spherical.

Although the second assumption renders the approach decentralized, the first assumption is a clear restriction to the decentralization level. We have therefore used the term "independent" instead of "decentralized" on the title of this paper.

We will now show that the system is asymptotically stabilized under the control law

$$
u_{i}=-K_{i} \frac{\partial \varphi_{i}}{\partial q_{i}}+\theta_{i}\left(v_{i}, \frac{\partial \varphi_{i}}{\partial t}\right)-g_{i} v_{i}
$$


where $K_{i}, g_{i}>0$ are positive gains,

$$
\theta_{i}\left(v_{i}, \frac{\partial \varphi_{i}}{\partial t}\right) \triangleq-\frac{c v_{i}}{\tanh \left(\left\|v_{i}\right\|^{2}\right)}\left|\frac{\partial \varphi_{i}}{\partial t}\right|
$$

and

$$
\frac{\partial \varphi_{i}}{\partial t}=\sum_{j \neq i} \frac{\partial \varphi_{i}}{\partial q_{j}} \dot{q}_{j}
$$

where $c>0$ is a positive scalar parameter. The first term of equation (3) corresponds to the decentralized navigation function described in the previous section to ensure collision avoidance of the multi-agent team. The second term exploits the knowledge each agent has of the velocities of the others, and is designed to guarantee convergence of the whole team to the desired configurations. The last term serves as a damping element that ensures convergence to the destination point by suppressing oscillatory motion around it.

By using the notation $x=\left[x_{1}^{T}, \ldots, x_{N}^{T}\right]^{T}, x_{i}^{T}=\left[\begin{array}{cc}q_{i}^{T} & v_{i}^{T}\end{array}\right]$ the closed loop dynamics of the system can be rewritten as

$$
\dot{x}=\xi(x)=\left[\xi_{1}^{T}(x), \ldots, \xi_{N}^{T}(x)\right]^{T}
$$

with

$$
\xi_{i}(x)=\left[\begin{array}{c}
v_{i} \\
-K_{i} \frac{\partial \varphi_{i}}{\partial q_{i}}-\frac{c v_{i}}{\tanh \left(\left\|v_{i}\right\|^{2}\right)}\left|\frac{\partial \varphi_{i}}{\partial t}\right|-g_{i} v_{i}
\end{array}\right]
$$

We will use the function $V=\sum_{i} K_{i} \varphi_{i}+\frac{1}{2} \sum_{i}\left\|v_{i}\right\|^{2}$ as a candidate Lyapunov function to show that the agents converge to their destinations points. We will check the stability of the multi-agent system with LaSalle's Invariance Principle. Specifically, the following theorem holds:

Theorem 3.1 System (4) is globally asymptotically stabilized to $\left[\begin{array}{ll}q_{d}^{T} & 0\end{array}\right], q_{d}=$ $\left[q_{d 1}, \ldots, q_{d N}\right]^{T}$ except from a set of initial conditions of measure zero if the exponent $k$ assumes values bigger than a finite lower bound and $c>\max _{i}\left(K_{i}\right)$. 
Proof: The candidate Lyapunov Function we use is $V=\sum_{i} K_{i} \varphi_{i}+\frac{1}{2} \sum_{i}\left\|v_{i}\right\|^{2}$ and by taking its derivative we have

$$
\begin{aligned}
& V=\sum_{i} K_{i} \varphi_{i}+\frac{1}{2} \sum_{i}\left\|v_{i}\right\|^{2} \Rightarrow \\
& \dot{V}=\sum K_{i} \dot{\varphi}_{i}+\sum v_{i}^{T} \dot{v}_{i}=\sum K_{i}\left(\frac{\partial \varphi_{i}}{\partial t}+v_{i}^{T} \frac{\partial \varphi_{i}}{\partial q_{i}}\right) \\
& +\sum v_{i}^{T}\left(-K_{i} \frac{\partial \varphi_{i}}{\partial q_{i}}+\theta_{i}\left(v_{i}, \frac{\partial \varphi_{i}}{\partial t}\right)-g_{i} v_{i}\right) \\
& \Rightarrow \dot{V}=\sum\left(K_{i} \frac{\partial \varphi_{i}}{\partial t}+v_{i}^{T} \theta_{i}\left(v_{i}, \frac{\partial \varphi_{i}}{\partial t}\right)-g_{i}\left\|v_{i}\right\|^{2}\right)
\end{aligned}
$$

Using the notation $B_{i} \triangleq K_{i} \frac{\partial \varphi_{i}}{\partial t}+v_{i}^{T} \theta_{i}\left(v_{i}, \frac{\partial \varphi_{i}}{\partial t}\right)$ we first show that $\sum_{i} B_{i} \leq 0$ if $c>\max _{i}\left(K_{i}\right)$ :

$$
\begin{aligned}
& \frac{\partial \varphi_{i}}{\partial t}>0: \\
& c>\max _{i}\left(K_{i}\right) \Rightarrow c>K_{i} \frac{\tanh \left(\left\|v_{i}\right\|^{2}\right)}{\left\|v_{i}\right\|^{2}} \\
& \Rightarrow K_{i}>\frac{c\left\|v_{i}\right\|^{2}}{\tanh \left(\left\|v_{i}\right\|^{2}\right)} \operatorname{sgn}\left(\frac{\partial \varphi_{i}}{\partial t}\right) \\
& \Rightarrow K_{i} \frac{\partial \varphi_{i}}{\partial t}+v_{i}^{T} \theta_{i}\left(v_{i}, \frac{\partial \varphi_{i}}{\partial t}\right)<0 \forall i: \frac{\partial \varphi_{i}}{\partial t}>0 \\
& \frac{\partial \varphi_{i}}{\partial t}<0: \\
& c>0 \Rightarrow c>-K_{i} \frac{\tanh \left(\left\|v_{i}\right\|^{2}\right)}{\left\|v_{i}\right\|^{2}} \\
& \Rightarrow K_{i}>\frac{c\left\|v_{i}\right\|^{2}}{\tanh \left(\left\|v_{i}\right\|^{2}\right)} \operatorname{sgn}\left(\frac{\partial \varphi_{i}}{\partial t}\right) \\
& \Rightarrow K_{i} \frac{\partial \varphi_{i}}{\partial t}+v_{i}^{T} \theta_{i}\left(v_{i}, \frac{\partial \varphi_{i}}{\partial t}\right)<0 \forall i: \frac{\partial \varphi_{i}}{\partial t}<0
\end{aligned}
$$

Of course, $K_{i} \frac{\partial \varphi_{i}}{\partial t}+v_{i}^{T} \theta_{i}\left(v_{i}, \frac{\partial \varphi_{i}}{\partial t}\right)=0$ for $\frac{\partial \varphi_{i}}{\partial t}=0$. In the preceding equations we used the fact that $0 \leq \frac{\tanh (x)}{x} \leq 1 \forall x \geq 0$. So we have $\sum_{i} B_{i} \leq 0$ with equality holding only when $\frac{\partial \varphi_{i}}{\partial t}=0 \forall i$. We have $\dot{V}=\sum_{i} B_{i}-\sum_{i} g_{i}\left\|v_{i}\right\|^{2} \leq 0$. Hence, by LaSalle's Invariance Principle, the state of the system converges to the largest invariant set contained in the set

$$
\begin{aligned}
& S=\left\{q, v:\left(\frac{\partial \varphi_{i}}{\partial t}=0\right) \wedge\left(v_{i}=0\right) \forall i\right\}= \\
& =\left\{q, v:\left(v_{i}=0\right) \forall i\right\}
\end{aligned}
$$

because by definition the set $\left\{q, v:\left(\frac{\partial \varphi_{i}}{\partial t}=0\right) \forall i\right\}$ is contained in the set $\left\{q, v:\left(v_{i}=0\right) \forall i\right\}$. For this subset to be invariant we need $\dot{v}_{i}=0 \Rightarrow \frac{\partial \varphi_{i}}{\partial q_{i}}=$ $0 \forall i$. 
The latter situation occurs whenever the potential functions either reach the destination or a saddle point. By bounding the parameter $k$ from below by a finite number, it is shown in [5] that the saddle points of $\varphi_{i}$ are isolated (following the recipe of [12]). Thus the set of initial conditions that lead to saddle points are sets of measure zero $([19])$. Hence the largest invariant set contained in the set $\frac{\partial \varphi_{i}}{\partial q_{i}}=0 \forall i$ is simply $q_{d}$.

$\diamond$

Hence, the control design of eq.(3) guarantees satisfaction of the desired specifications, namely collision avoidance and convergence to the destination of the multi-agent team.

\section{Nonholonomic Dynamics}

In [17], the decentralized navigation functions methodology has been extended for the case of multiple agents with nonholonomic kinematics. Although each agent had no specific knowledge about the destinations of the others, it treated a spherical region around the target of each agent as a static obstacle. In this section we modify the proposed control law in order to allow each agent to neglect the destinations of the others. Furthermore, the control inputs are the acceleration and rotational velocity of each vehicle, coping in this way with realistic classes of mechanical systems.

Let us consider the situation described at the beginning of section 2 (see also fig.1). Each of the $N$ mobile agents has a specific orientation $\theta_{i}$ with respect to a global coordinate frame. The orientation vector of the agents is represented by $\theta=\left[\theta_{1} \ldots \theta_{N}\right]$. The configuration of each agent is represented by $p_{i}=\left[\begin{array}{ll}q_{i} & \theta_{i}\end{array}\right] \in \mathbb{R}^{2} \times(-\pi, \pi]$ and it's target by $p_{d i}=\left[\begin{array}{cc}q_{d i} & \theta_{d i}\end{array}\right] \in$ $\mathbb{R}^{2} \times(-\pi, \pi]$. Hence the goal configuration of each agent includes both a desired final position and a desired final orientation.

The nonholonomic kinematics and simplified dynamics of the agents are 
given by the following combination of a unicycle model and a single integrator

$$
\begin{aligned}
& \dot{x}_{i}=v_{i} \cos \theta_{i} \\
& \dot{y}_{i}=v_{i} \sin \theta_{i} \\
& \dot{\theta}_{i}=\omega_{i} \\
& \dot{v}_{i}=u_{i}
\end{aligned}
$$

where $v_{i}, \omega_{i}$ are the translational and rotational velocities of agent $i$ respectively, and $u_{i}$ its acceleration.

The problem we treat in this section can be now stated as follows: "Given the $N$ nonholonomic agents (5), consider the rotational velocity $\omega_{i}$ and the acceleration $u_{i}$ as control inputs for each agent and derive a control law that steers every agent from any feasible initial configuration to its goal configuration avoiding, at the same, collisions."

We make the following assumptions:

- Each agent has global knowledge of the position of the others at each time instant.

- Each agent has knowledge only of its own desired destination but not of the others.

- Agents are assumed to be spherical.

- The workspace is bounded and spherical.

\subsection{Decentralized Dipolar Navigation Functions}

It was shown in [5] that the function: $\varphi_{i}=\frac{\gamma_{d i}+f_{i}}{\left(\left(\gamma_{d i}+f_{i}\right)^{k}+G_{i}\right)^{1 / k}}$ with a proper selection of $G_{i}$ can be used for decentralized motion planning of multiple holonomic robots and can be made a navigation function by an appropriate choice of $k$. In this section we present how this class of potential functions 
can be enhanced with a dipolar structure [25] to provide trajectories suitable for nonholonomic navigation.

Dipolar potential fields have been proven a very effective tool for stabilization [26] of nonholonomic systems as well as for centralized coordination of multiple agents with nonholonomic constraints [18]. The key advantage of this class of potential fields is that they drive the controlled agent to its destination with desired orientation.

To be able to produce a dipolar potential field, $\varphi_{i}$ is redefined as:

$$
\varphi_{i}=\frac{\gamma_{d i}+f_{i}}{\left(\left(\gamma_{d i}+f_{i}\right)^{k}+H_{n h_{i}} \cdot G_{i}\right)^{1 / k}}
$$

where $H_{n h_{i}}$ represents a pseudo - obstacle that ensures that the navigation function (6) assumes the form of a dipolar filed. A possible selection of $H_{n h_{i}}$ is:

$$
H_{n h_{i}}=\varepsilon_{n h}+\eta_{n h_{i}}
$$

with $\eta_{n h_{i}}=\left\|\left(q_{i}-q_{d i}\right) \cdot \eta_{d_{i}}\right\|^{2}$, where $\eta_{d_{i}}=\left[\cos \left(\theta_{d_{i}}\right) \sin \left(\theta_{d_{i}}\right)\right]^{T}$. Moreover $\gamma_{d i}=\left\|q_{i}-q_{d_{i}}\right\|^{2}$, i.e. the angle is not incorporated in the distance to the destination metric. This choice of $\varphi_{i}$ has been used in [17] for the case of nonholonomic kinematics. In this case the $f_{i}$ function has been included in order cope with the requirement that each agent is unaware of the desired destinations of the others. The scalar positive parameter $\varepsilon_{n h}>0$ is chosen to ensure that the proposed modification of the potential function does not affect its navigation properties([17],[18]). Figure 2 shows a plot of the dipolar navigation function $\varphi_{i}$.

In order to proceed with the control design and stability analysis for the system in hand, one has to consider a few results from nonsmooth analysis and Lyapunov theory for nonsmooth systems found in appendix A. 


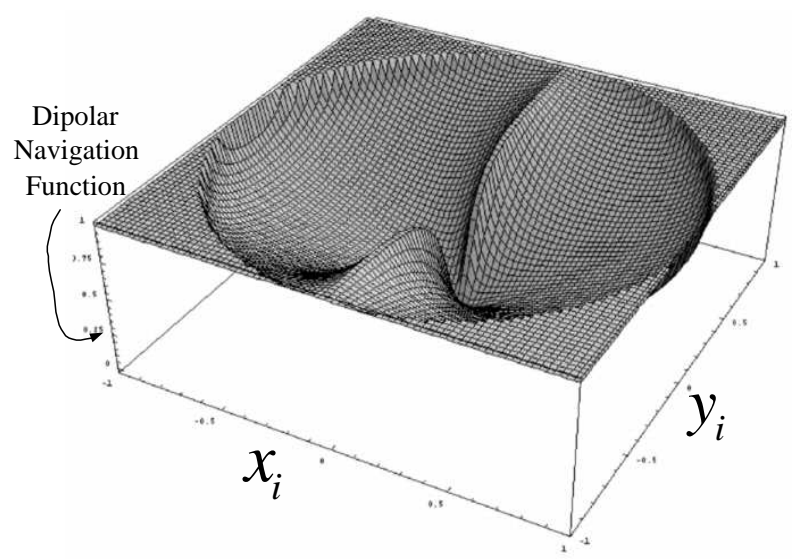

Figure 2: 2D dipolar navigation function

\subsection{Nonholonomic Control and Stability Analysis}

We will show that the system is asymptotically stabilized under the control law

$$
\begin{gathered}
u_{i}=-v_{i}\left\{\left|\nabla_{i} \varphi_{i} \cdot \eta_{i}\right|+M_{i}\right\}-g_{i} v_{i}-\frac{v_{i}}{\tanh \left(\left|v_{i}\right|\right)} K_{v_{i}} K_{z_{i}} \\
\omega_{i}=-K_{\theta_{i}}\left(\theta_{i}-\theta_{d i}-\theta_{n h_{i}}\right)+\dot{\theta}_{n h_{i}}
\end{gathered}
$$

where $K_{v_{i}}, K_{\theta_{i}}, g_{i}>0$ are positive gains, $\theta_{n h_{i}}=\arg \left(\frac{\partial \varphi_{i}}{\partial x_{i}} \cdot s_{i}+\mathbf{i} \frac{\partial \varphi_{i}}{\partial y_{i}} \cdot s_{i}\right), s_{i}=$ $\operatorname{sgn}\left(\left(q_{i}-q_{d i}\right) \cdot \eta_{d i}\right), \eta_{i}=\left[\begin{array}{ll}\cos \theta_{i} & \sin \theta_{i}\end{array}\right]^{T}, \eta_{d i}=\left[\begin{array}{cc}\cos \theta_{d i} & \sin \theta_{d i}\end{array}\right]^{T}, K_{z i}=$ $\left\|\nabla_{i} \varphi_{i}\right\|^{2}+\left\|q_{i}-q_{d i}\right\|^{2}, M_{i}>\left|\sum_{j \neq i} \nabla_{i} \varphi_{j} \cdot \eta_{i}\right|_{\max }$ and $\nabla_{i} \varphi_{j}=\left[\frac{\partial \varphi_{j}}{x_{i}} \frac{\partial \varphi_{j}}{y_{i}}\right]$. In particular, we prove the following theorem:

Theorem 4.1 Under the control law (7), the system is asymptotically stabilized to $p_{d}=\left[p_{d 1}, \ldots, p_{d N}\right]^{T}$.

Proof: Let us first consider the case $\left|v_{i}\right|>0 \forall i$. We use

$$
V=\sum V_{i}, V_{i}=\varphi_{i}+\left|v_{i}\right|+\frac{1}{2}\left(\theta_{i}-\theta_{d i}-\theta_{n h i}\right)^{2}
$$


as a Lyapunov function candidate. For $\left|v_{i}\right|>0$ we have

$$
\dot{V}=\sum_{i} \dot{V}_{i}=\sum_{i}\left\{\begin{array}{c}
\sum_{j} v_{j}\left(\nabla_{j} \varphi_{i}\right) \cdot \eta_{j}+\operatorname{sgn}\left(v_{i}\right) \dot{v}_{i}+ \\
+\left(\theta_{i}-\theta_{d i}-\theta_{n h i}\right)\left(\dot{\theta}_{i}-\dot{\theta}_{n h i}\right)
\end{array}\right\}
$$

and substituting

$$
\begin{aligned}
& \dot{V}=\sum_{i}\left\{\sum_{j} v_{j}\left(\nabla_{j} \varphi_{i}\right) \cdot \eta_{j}-\left|v_{i}\right|\left(\left|\left(\nabla_{i} \varphi_{i}\right) \cdot \eta_{i}\right|+M_{i}\right)\right\} \\
& -\sum_{i} \frac{\left|v_{i}\right|}{\tanh \left(\left|v_{i}\right|\right)} K_{v_{i}} K_{z_{i}}-\sum_{i} g_{i}\left|v_{i}\right| \\
& -\sum_{i} K_{\theta_{i}}\left(\theta_{i}-\theta_{d i}-\theta_{n h i}\right)^{2}
\end{aligned}
$$

The first term of the right hand side of the last equation can be rewritten as

$$
\begin{aligned}
& \sum_{i}\left\{\sum_{j} v_{j}\left(\nabla_{j} \varphi_{i}\right) \cdot \eta_{j}-\left|v_{i}\right|\left(\left|\left(\nabla_{i} \varphi_{i}\right) \cdot \eta_{i}\right|+M_{i}\right)\right\}= \\
& =\sum_{i}\left\{\begin{array}{l}
v_{i}\left(\nabla_{i} \varphi_{i}\right) \cdot \eta_{i}+v_{i} \sum_{j \neq i}\left(\nabla_{i} \varphi_{j}\right) \cdot \eta_{i}- \\
-\left|v_{i}\right|\left(\left|\left(\nabla_{i} \varphi_{i}\right) \cdot \eta_{i}\right|+M_{i}\right)
\end{array}\right\} \leq 0
\end{aligned}
$$

so that $\dot{V} \leq-\sum_{i} K_{v_{i}} K_{z_{i}}-\sum_{i} g_{i}\left|v_{i}\right|-\sum_{i} K_{\theta_{i}}\left(\theta_{i}-\theta_{d i}-\theta_{n h i}\right)^{2}$ where the inequality $\frac{x}{\tanh x} \geq 1$ for $x \geq 0$.

The candidate Lyapunov function is nonsmooth whenever $v_{i}=0$ for some $i$. The generalized gradient of $V$ and the Filippov set of the closed loop system by are respectively given by 


$$
\partial V=\left[\begin{array}{c}
\sum_{i} \nabla_{1} \varphi_{i} \\
\vdots \\
\sum_{i} \nabla_{N} \varphi_{i} \\
\partial\left|v_{1}\right| \\
\vdots \\
\partial\left|v_{N}\right| \\
\frac{1}{2} \nabla_{\theta_{1}}\left(\theta_{1}-\theta_{d 1}-\theta_{n h 1}\right)^{2} \\
\vdots \\
\frac{1}{2} \nabla_{\theta_{N}}\left(\theta_{N}-\theta_{d N}-\theta_{n h N}\right)^{2} \\
\frac{1}{2} \nabla_{\theta_{n h 1}}\left(\theta_{1}-\theta_{d 1}-\theta_{n h 1}\right)^{2} \\
\vdots \\
\frac{1}{2} \nabla_{\theta_{n h N}}\left(\theta_{N}-\theta_{d N}-\theta_{n h N}\right)^{2}
\end{array}\right],\left[\begin{array}{c}
v_{1} \cos \theta_{1} \\
v_{1} \sin \theta_{1} \\
\vdots \\
v_{N} \cos \theta_{N} \\
v_{N} \sin \theta_{N} \\
u_{1} \\
\vdots \\
u_{N} \\
\omega_{1} \\
\vdots \\
\omega_{N} \\
\dot{\theta}_{n h 1} \\
\vdots \\
\dot{\theta}_{n h N}
\end{array}\right]=\left[\begin{array}{c}
v_{1} \cos \theta_{1} \\
v_{1} \sin \theta_{1} \\
\vdots \\
v_{N} \cos \theta_{N} \\
v_{N} \sin \theta_{N} \\
K\left[u_{1}\right] \\
\vdots \\
K\left[u_{N}\right] \\
\omega_{1} \\
\vdots \\
\omega_{N} \\
\dot{\theta}_{n h 1} \\
\vdots \\
\dot{\theta}_{n h N}
\end{array}\right]
$$

We denote by $D \triangleq\left\{x: \exists i \in\{1, \ldots N\}\right.$ s.t. $\left.v_{i}=0\right\}$ the "discontinuity surface" and $D_{S} \triangleq\left\{i \in\{1, \ldots N\}\right.$ s.t. $\left.v_{i}=0\right\}$ the set of indices of agents that participate in $D$. We then have

$$
\begin{aligned}
& \dot{\vec{V}}=\bigcap_{\xi \in \partial V} \xi^{T} K[f]= \\
& v_{1}\left(\sum_{i} \nabla_{1} \varphi_{i}\right) \cdot \eta_{1}+\ldots+v_{N}\left(\sum_{i} \nabla_{N} \varphi_{i}\right) \cdot \eta_{N} \\
& +\bigcap_{\xi \in \partial\left|v_{1}\right|} \xi^{T} K\left[u_{1}\right]+\ldots+\bigcap_{\xi \in \partial\left|v_{N}\right|} \xi^{T} K\left[u_{N}\right] \\
& +\sum_{i}\left(\theta_{i}-\theta_{d i}-\theta_{n h i}\right)\left(\omega_{i}-\dot{\theta}_{n h i}\right) \Rightarrow \\
& \dot{\tilde{V}}=\sum_{i \notin D_{S}}\left\{v_{i}\left(\sum_{i} \nabla_{i} \varphi_{j}\right) \cdot \eta_{i}+\operatorname{sgn}\left(v_{i}\right) u_{i}\right\} \\
& +\sum_{i \in D_{S}} \bigcap_{\xi \in \partial\left|v_{i}\right|} \xi^{T} K\left[u_{i}\right]-\sum_{i} K_{\theta_{i}}\left(\theta_{i}-\theta_{d i}-\theta_{n h i}\right)^{2}
\end{aligned}
$$


For $i \in D_{S}$ we have $\partial\left|v_{i}\right|_{v_{i}=0}=[-1,1]$ and $\left.K\left[u_{i}\right]\right|_{v_{i}=0}=\left[-\left|K_{v i} K_{z i}\right|,\left|K_{v i} K_{z i}\right|\right]$ so that $\bigcap_{\xi \in \partial\left|v_{i}\right|} \xi^{T} K\left[u_{i}\right]=0$. From the previous analysis we also derive that

$$
\begin{aligned}
& \sum_{i \notin D_{S}}\left\{v_{i}\left(\sum_{i} \nabla_{i} \varphi_{j}\right) \cdot \eta_{i}+\operatorname{sgn}\left(v_{i}\right) u_{i}\right\} \leq \\
& -\sum_{i \notin D_{S}}\left\{K_{v i} K_{z i}+g_{i}\left|v_{i}\right|\right\}
\end{aligned}
$$

Going back to Theorem A.2 it is easy to see that $v \leq 0 \forall v \in \dot{\widetilde{V}}$. Each function $V_{i}$ is regular as the sum of regular functions ([24]) and $V$ is regular for the same reason. The level sets of $V$ are compact so we can apply this theorem. We have that $S=\{x \mid 0 \in \dot{\widetilde{V}}\}=\left\{x:\left(v_{i}=0 \forall i\right) \bigwedge\left(\theta_{i}-\theta_{d i}=\theta_{n h i} \forall i\right)\right\}$. The trajectory of the system converges to the largest invariant subset of $S$. For this subset to be invariant we must have

$$
\dot{v}_{i}=0 \Rightarrow K_{v i} K_{z i}=0 \Rightarrow\left(\nabla_{i} \varphi_{i}=0\right) \wedge\left(q_{i}=q_{d i}\right) \forall i
$$

For $\nabla_{i} \varphi_{i}=0$ we have $\theta_{n h i}=0$ so that $\theta_{i}=\theta_{d i}$.

$\diamond$

This establishes that each nonholonomic agent converges to its goal configuration with the desired orientation.

\section{Simulations}

To demonstrate the navigation properties of our decentralized approach, we present a series of simulations of multiple agents that have to navigate from an initial to a final configuration, avoiding collision with each other. The chosen configurations constitute non-trivial setups since the straight-line paths connecting initial and final positions of each agent are obstructed by other agents. 
The navigation properties of the proposed control scheme are verified in the dynamic case as well through the non-trivial simulations in the following two figures involving four holonomic and nonholonomic agents respectively.

Figure 5 involves four holonomic agents with double integrator dynamics navigating under the control law (3). Screenshots A through $\mathrm{F}$ show the evolution of the four agent team. In screenshot A, $I-i, T-i$ denote the initial position and final desired destination of agent $i$ respectively. The conflict resolution procedure takes place in screenshots B through D. In the last to screenshots, agents converge to their final configurations, free of collisions. The parameters chosen for this simulation are:

\section{Initial Conditions:}

$$
\begin{gathered}
q_{1}(0)=\left[\begin{array}{ll}
.1232 & -.1
\end{array}\right]^{T}, q_{2}(0)=\left[\begin{array}{ll}
-.1 & -.1
\end{array}\right]^{T}, \\
q_{3}(0)=\left[\begin{array}{ll}
-.1232 & .1
\end{array}\right]^{T}, q_{4}(0)=\left[\begin{array}{ll}
.1 & .1
\end{array}\right]^{T} \\
u_{1}(0)=u_{2}(0)=u_{3}(0)=u_{4}(0)=\left[\begin{array}{ll}
10^{-3} & 0
\end{array}\right]
\end{gathered}
$$

Final Conditions:

$$
\begin{aligned}
& q_{d 1}=\left[\begin{array}{ll}
-.1232 & .1
\end{array}\right]^{T}, q_{d 2}=\left[\begin{array}{ll}
.1 & .1
\end{array}\right]^{T}, \\
& q_{d 3}=\left[\begin{array}{ll}
.1732 & -.1
\end{array}\right]^{T}, q_{d 4}=\left[\begin{array}{ll}
-.1 & -.1
\end{array}\right]^{T}
\end{aligned}
$$

Parameters:

$$
r_{1}=r_{2}=r_{3}=r_{4}=0.04, k=80
$$

where without loss of generality we used the same gains $K_{i}=K$ and $g_{i}=g$ for each $i=1,2,3,4$.

Figure 6 involves four nonholonomic agents with dynamics described by 5 navigating under the control law (7). Screenshots A through $\mathrm{H}$ show the evolution of the four agent team. In screenshot $\mathrm{A}, I-i, T-i$ denote the initial position and final desired destination of agent $i$ respectively. The conflict resolution procedure takes place in screenshots $\mathrm{C}$ through $\mathrm{F}$, while 

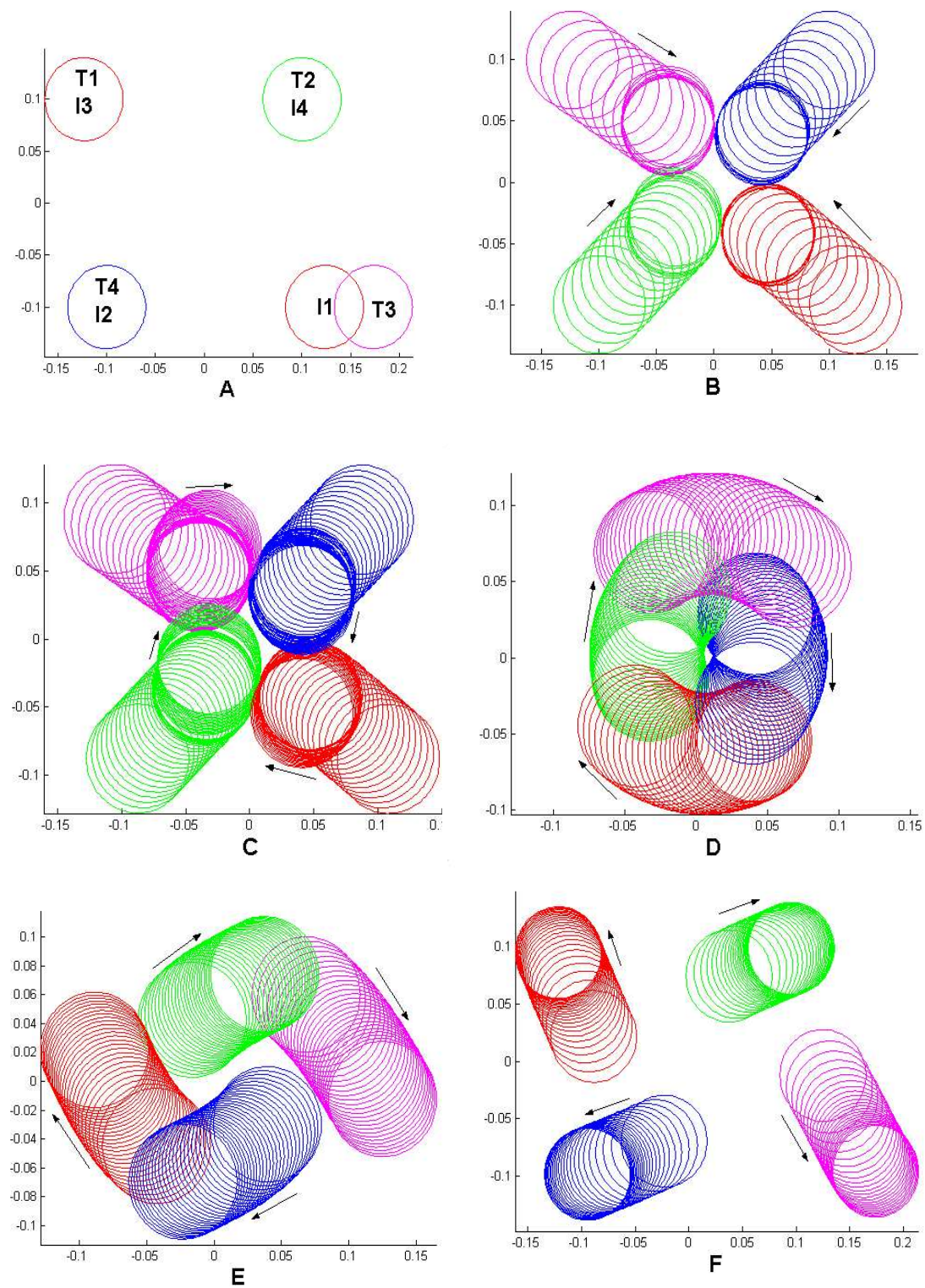

Figure 3: 4 dynamic holonomic agents 
in the last two screenshots the agents converge to their goal configuration free of collisions. The parameters chosen for this simulation are:

Initial Conditions:

$$
\begin{aligned}
& p_{1}(0)= {\left[\begin{array}{lll}
-.1232 & .1 & 0
\end{array}\right]^{T}, p_{2}(0)=\left[\begin{array}{lll}
0 & 0 & \pi
\end{array}\right]^{T}, } \\
& p_{3}(0)=\left[\begin{array}{lll}
-.1232 & -.1232 & \pi / 4
\end{array}\right]^{T}, p_{4}(0)=\left[\begin{array}{lll}
.1232 & -.1 & 0
\end{array}\right]^{T} \\
& u_{1}(0)=u_{2}(0)=u_{3}(0)=u_{4}(0)=\left[\begin{array}{ll}
10^{-3} & 0
\end{array}\right]
\end{aligned}
$$

Final Conditions:

$$
\begin{aligned}
& p_{d 1}=\left[\begin{array}{lll}
-.1232 & .1 & 0
\end{array}\right]^{T}, p_{d 2}=\left[\begin{array}{lll}
.1 & .1 & 0
\end{array}\right]^{T}, \\
& p_{d 3}=\left[\begin{array}{lll}
.1732 & -.1 & 0
\end{array}\right]^{T}, p_{d 4}=\left[\begin{array}{lll}
-.1 & -.1 & 0
\end{array}\right]^{T}
\end{aligned}
$$

Parameters:

$$
r_{1}=r_{2}=r_{3}=r_{4}=0.01, \varepsilon_{n h}=10^{-5}, k=90, M=g=K_{v}=10^{-3}, K_{\theta}=1
$$

The collision avoidance procedure shown in screenshots $\mathrm{C}$ through $\mathrm{F}$ reveals the cooperative nature of our approach. As seen in screenshots C,D agents 1,2 backtrack from their desired destination to create free space for agents 3,4 to navigate towards their desired destinations. The conflict resolution maneuvers of screenshots E,F allow agents 1,2 to find free space and converge to their goal configuration.

\section{Conclusion}

A decentralized multi-agent navigation functions' methodology, established in previous work for multi-agent systems with kinematic models of motion, has been extended to the case of dynamic models of motion both for holonomic and nonholonomic agents. The proposed control scheme guarantees collision avoidance and global convergence of the multi-agent team to the 


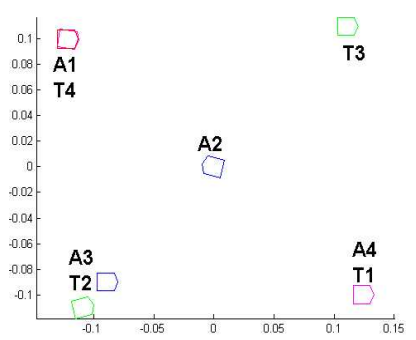

A
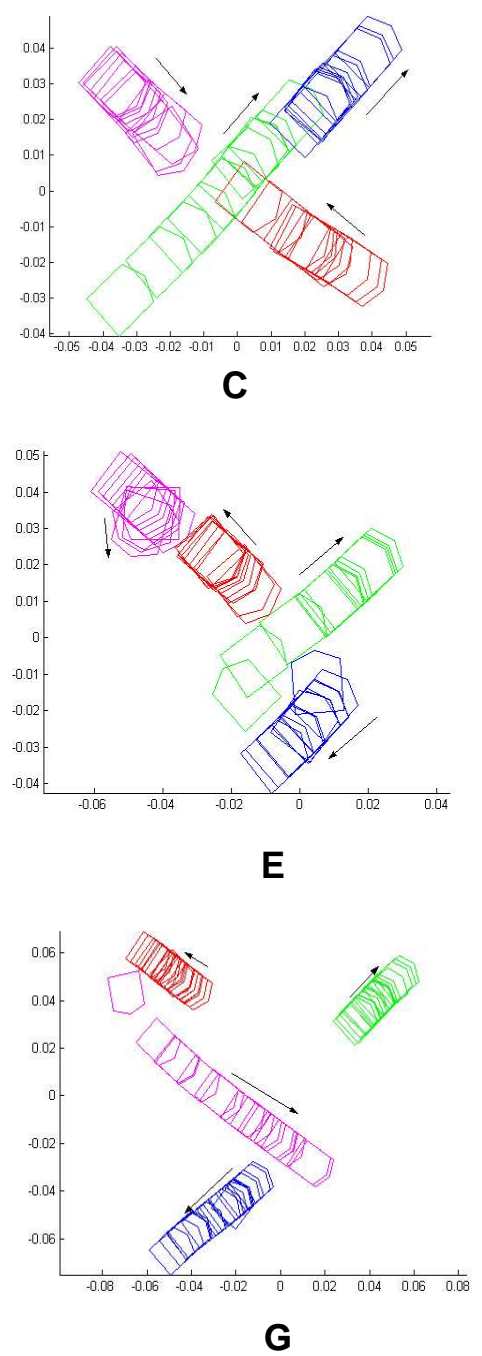

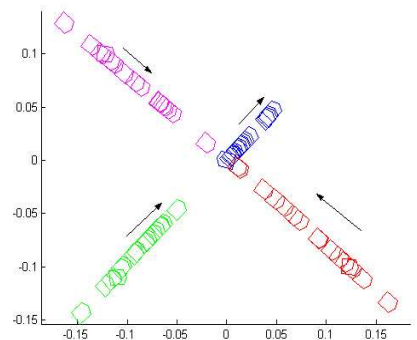

B
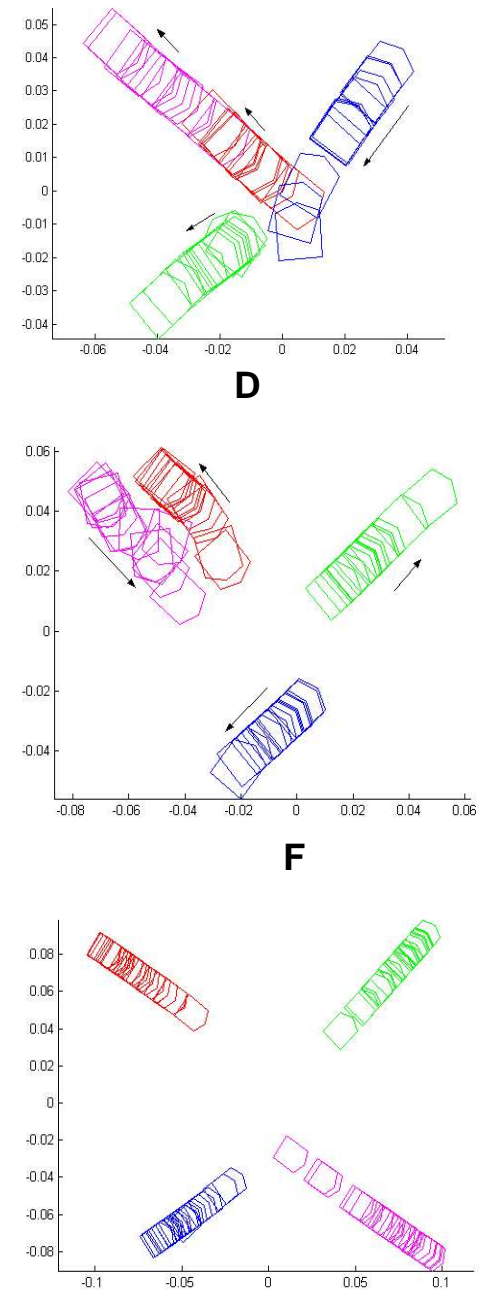

H

Figure 4: 4 dynamic nonholonomic agents 
desired goal configuration. The volume of each agent has been taken into account and non-point models have been considered. The satisfaction of the imposed system specifications under the proposed control scheme have been verified through non-trivial computer simulations.

Current research aims at considering the above issues for the case of limited sensing capabilities of each agent about the rest of the team,i.e. increasing the decentralization of the whole scheme. This has been accomplished for the case of single integrator dynamics in a recent paper ([4]). Another interesting issue that has to be dealt is consideration of three-dimensional dynamic models of vehicle motion.

\section{A Elements from Nonsmooth Analysis}

In this section, we review some elements from nonsmooth analysis and Lyapunov theory for nonsmooth systems that we use in the stability analysis of the next section.

We consider the vector differential equation with discontinuous right-hand side:

$$
\dot{x}=f(x)
$$

where $f: R^{n} \rightarrow R^{n}$ is measurable and essentially locally bounded.

Definition A.1 [9]: In the case when $n$ is finite, the vector function $x($.$) is$ called a solution of (8) in $\left[t_{0}, t_{1}\right]$ if it is absolutely continuous on $\left[t_{0}, t_{1}\right]$ and there exists $N_{f} \subset R^{n}, \mu\left(N_{f}\right)=0$ such that for all $N \subset R^{n}, \mu(N)=0$ and for almost all $t \in\left[t_{0}, t_{1}\right]$

$$
\dot{x} \in K[f](x) \equiv \overline{c o}\left\{\lim _{x_{i} \rightarrow x} f\left(x_{i}\right) \mid x_{i} \notin N_{f} \cup N\right\}
$$

Lyapunov stability theorems have been extended for nonsmooth systems in [24],[1]. The authors use the concept of generalized gradient which for the case of finite-dimensional spaces is given by the following definition: 
Definition A.2 [2]: Let $V: R^{n} \rightarrow R$ be a locally Lipschitz function. The generalized gradient of $V$ at $x$ is given by

$$
\partial V(x)=\overline{c o}\left\{\lim _{x_{i} \rightarrow x} \nabla V\left(x_{i}\right) \mid x_{i} \notin \Omega_{V}\right\}
$$

where $\Omega_{V}$ is the set of points in $R^{n}$ where $V$ fails to be differentiable.

Lyapunov theorems for nonsmooth systems require the energy function to be regular. Regularity is based on the concept of generalized derivative which was defined by Clarke as follows:

Definition A.3 [2]: Let $f$ be Lipschitz near $x$ and $v$ be a vector in $R^{n}$. The generalized directional derivative of $f$ at $x$ in the direction $v$ is defined

$$
f^{0}(x ; v)=\lim _{y \rightarrow x} \sup _{t \downarrow 0} \frac{f(y+t v)-f(y)}{t}
$$

Definition A.4 [2]: The function $f: R^{n} \rightarrow R$ is called regular if 1) $\forall v$, the usual one-sided directional derivative $f^{\prime}(x ; v)$ exists and 2) $\forall v, f^{\prime}(x ; v)=f^{0}(x ; v)$

The following chain rule provides a calculus for the time derivative of the energy function in the nonsmooth case:

Theorem A.1 [24]: Let $x$ be a Filippov solution to $\dot{x}=f(x)$ on an interval containing $t$ and $V: R^{n} \rightarrow R$ be a Lipschitz and regular function. Then $V(x(t))$ is absolutely continuous, $(d / d t) V(x(t))$ exists almost everywhere and

$$
\frac{d}{d t} V(x(t)) \in^{a . e \cdot} \dot{\widetilde{V}}(x):=\bigcap_{\xi \in \partial V(x(t))} \xi^{T} K[f](x(t))
$$

We shall use the following nonsmooth version of LaSalle's invariance principle to prove the convergence of the prescribed system: 
Theorem A.2 [24] Let $\Omega$ be a compact set such that every Filippov solution to the autonomous system $\dot{x}=f(x), x(0)=x\left(t_{0}\right)$ starting in $\Omega$ is unique and remains in $\Omega$ for all $t \geq t_{0}$. Let $V: \Omega \rightarrow R$ be a time independent regular function such that $v \leq 0 \forall v \in \dot{\widetilde{V}}$ (if $\dot{\vec{V}}$ is the empty set then this is trivially satisfied). Define $S=\{x \in \Omega \mid 0 \in \dot{\widetilde{V}}\}$. Then every trajectory in $\Omega$ converges to the largest invariant set, $M$, in the closure of $S$.

\section{References}

[1] F. Ceragioli. Discontinuous Ordinary Differential Equations and Stabilization. PhD thesis, Dept. of Mathematics, Universita di Firenze, 1999.

[2] F. Clarke. Optimization and Nonsmooth Analysis. Addison - Wesley, 1983.

[3] J. Cortes, S. Martinez, and F. Bullo. Robust rendezvous for mobile autonomous agents via proximity graphs in d dimensions. IEEE Transactions on Automatic Control, submitted for publication, 2004.

[4] D. V. Dimarogonas and K. J. Kyriakopoulos. Decentralized stabilization and collision avoidance of multiple air vehicles with limited sensing capabilities. 2005 American Control Conference, pages 4667-4772.

[5] D. V. Dimarogonas, S. G. Loizou, K.J. Kyriakopoulos, and M. M. Zavlanos. A feedback stabilization and collision avoidance scheme for multiple independent non-point agents. Automatica, 42(2):229-243, 2006.

[6] D.V. Dimarogonas and K.J. Kyriakopoulos. Decentralized motion control of multiple agents with double integrator dynamics. 16th IFAC World Congress, 2005. 
[7] D.V. Dimarogonas and K.J. Kyriakopoulos. A feedback stabilization and collision avoidance scheme for multiple independent nonholonomic non-point agents. Joint International Symposium on Intelligent Control E 13 th Mediterranean Conference on Control and Automation, pages 820-825, 2005.

[8] D.V. Dimarogonas, M.M. Zavlanos, S.G. Loizou, and K.J. Kyriakopoulos. Decentralized motion control of multiple holonomic agents under input constraints. 42nd IEEE Conference on Decision and Control, pages 3390-3395, 2003.

[9] A. Filippov. Differential equations with discontinuous right-hand sides. Kluwer Academic Publishers, 1988.

[10] V. Gazi and K.M. Passino. Stability analysis of swarms. IEEE Transactions on Automatic Control, 48(4):692-696, 2003.

[11] M. Ji and M. Egerstedt. Connectedness preserving distibuted coordination control over dynamic graphs. 2005 American Control Conference, pages $93-98$.

[12] D. E. Koditschek and E. Rimon. Robot navigation functions on manifolds with boundary. Advances Appl. Math., 11:412-442, 1990.

[13] G. Lafferriere, A. Williams, J. Caughman, and J.J.P. Veerman. Decentralized control of vehicle formations. Systems and Control Letters, 54(9):899-910, 2005.

[14] J. Lin, A.S. Morse, and B. D. O. Anderson. The multi-agent rendezvous problem. 42st IEEE Conf. Decision and Control, pages 1508-1513, 2003.

[15] Z. Lin, B. Francis, and M. Maggiore. Necessary and sufficient graphical conditions for formation control of unicycles. IEEE Transactions on Automatic Control, 50(1):121-127, 2005. 
[16] S. G. Loizou and K. J. Kyriakopoulos. Closed loop navigation for multiple holonomic vehicles. Proc. of IEEE/RSJ Int. Conf. on Intelligent Robots and Systems, pages 2861-2866, 2002.

[17] S.G. Loizou, D.V. Dimarogonas, and K.J. Kyriakopoulos. Decentralized feedback stabilization of multiple nonholonomic agents. 2004 IEEE International Conference on Robotics and Automation, pages 3012-3017.

[18] S.G. Loizou and K.J. Kyriakopoulos. Closed loop navigation for multiple non-holonomic vehicles. IEEE Int. Conf. on Robotics and Automation, pages 420-425, 2003.

[19] J. Milnor. Morse theory. Annals of Mathematics Studies. Princeton University Press, Princeton, NJ, 1963.

[20] L. Moreau. Stability of continuous-time distributed consensus algorithms. 43rd IEEE Conf. Decision and Control, pages 3998-4003, 2004.

[21] R. Olfati-Saber. Flocking for multi-agent dynamic systems: Algorithms and theory. IEEE Transactions on Automatic Control, 51(3):in press, 2006.

[22] L. Pallottino, V.G. Scordio, E. Frazzoli, and A. Bicchi. Probabilistic verification of a decentralized policy for conflict resolution in multi-agent systems. IEEE Intern. Conf. Robotics and Automation, 2006.

[23] W. Ren and R. Beard. Consensus seeking in multiagent systems under dynamically changing interaction topologies. IEEE Transactions on Automatic Control, 50(5):655-661, 2005.

[24] D. Shevitz and B. Paden. Lyapunov stability theory of nonsmooth systems. IEEE Trans. on Automatic Control, 49(9):1910-1914, 1994. 
[25] H. G. Tanner and K. J. Kyriakopoulos. Nonholonomic motion planning for mobile manipulators. Proc of IEEE Int. Conf. on Robotics and Automation, pages 1233-1238, 2000.

[26] H. G. Tanner, S. G. Loizou, and K. J. Kyriakopoulos. Nonholonomic navigation and control of cooperating mobile manipulators. IEEE Trans. on Robotics and Automation, 19(1):53-64, 2003.

[27] H.G. Tanner, A. Jadbabaie, and G.J. Pappas. Stable flocking of mobile agents. 42st IEEE Conf. Decision and Control, 2003.

[28] H.G. Tanner and A. Kumar. Formation stabilization of multiple agents using decentralized navigation functions. Robotics: Science and Systems, 2005.

[29] M.M. Zavlanos and K.J. Kyriakopoulos. Decentralized motion control of multiple mobile agents. 11th Mediterranean Conference on Control and Automation, 2003. 